\title{
Influences of Reduced Expression of Maternal Bone Morphogenetic Protein 2 on Mouse Embryonic Development
}

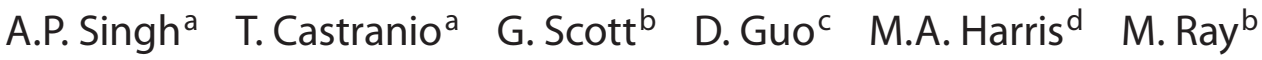 \\ S.E. Harris ${ }^{d} \quad$ Y. Mishina ${ }^{a, b}$ \\ ${ }^{a}$ Molecular Developmental Biology Group, Laboratory of Reproductive and Developmental Toxicology, \\ ${ }^{b}$ Knockout Mouse Core, National Institute of Environmental Health Sciences, National Institutes of Health, \\ Research Triangle Park, N.C., 'University of Missouri at Kansas City, Kansas City, Mo., ' University of Texas \\ Health Science Center at San Antonio, San Antonio, Tex., USA
}

\section{Key Words}

BMP2 $\cdot$ Decidualization $\cdot$ Hypomorphic $\cdot$ Neural tube

closure $\cdot$ Omphalocele

\begin{abstract}
Bone morphogenetic protein 2 (BMP2) was originally found by its osteoinductive ability, and recent genetic analyses have revealed that it plays critical roles during early embryogenesis, cardiogenesis, decidualization as well as skeletogenesis. In the course of evaluation of the conditional allele for Bmp2, we found that the presence of a neo cassette, a selection marker needed for gene targeting events in embryonic stem cells, in the 3' untranslated region of exon 3 of $B m p 2$, reduced the expression levels of Bmp2 both in embryonic and maternal mouse tissues. Some of the embryos that were genotyped as transheterozygous for the floxed allele with the neo cassette over the conventional null allele ( $f n /-)$ showed a lethal phenotype including defects in cephalic neural tube closure and ventral abdominal wall closure. The number of embryos exhibiting these abnormalities was increased when, due to different genotypes, expression levels of Bmp2 in maternal tissues were lower. These results suggest that the expression levels of Bmp2 in both embryonic and maternal tissues influence the normal neural tube closure and body wall closure with different thresholds.
\end{abstract}

Copyright $\odot 2008$ S. Karger AG, Basel

\section{KARGER}

Fax +4161306 1234 E-Mail karger@karger.ch www.karger.com
(C) 2008 S. Karger AG, Basel

$1661-5425 / 08 / 0023-0134 \$ 24.50 / 0$

Accessible online at:

www.karger.com/sxd
TGF- $\beta$ superfamily members are important for embryogenesis, cell growth and differentiation including sexual differentiation [de Caestecker, 2004]. Bone morphogenetic proteins (BMPs) are a subfamily of the TGF- $\beta$ superfamily that were originally identified due to their osteoinductive ability but play various roles during development [Kishigami and Mishina, 2005]. BMP2 was one of the first members to be identified and its conventional gene disruption results in early embryonic lethality soon after gastrulation [Zhang and Bradley, 1996]. Using this mutant null (-) allele in heterozygotes (+/-) revealed that BMP2 is a critical factor for the migration but not induction of neural crest cells [Kanzler et al., 2000; Correia et al., 2007].

To overcome the embryonic lethality, floxed mouse lines for Bmp2 have recently become available, and BMP2 functions in the later stages of development, including cardiogenesis and skeletogenesis, have begun to emerge [Ma et al., 2005; Tsuji et al., 2006]. Recently, it has been reported that a uterine-specific disruption of $B m p 2$ leads to failure of decidualization causing abortion of embryos [Lee et al., 2007]. We independently developed a conditional allele of $B m p 2$ (manuscript in preparation). Besides two loxP sites for conditional removal of exon 3 , we inserted a neo resistant cassette driven by a polII promoter flanked by FRT sites into the $3^{\prime}$-untranslated region

Molecular Developmental Biology Group, Laboratory of Reproductive and Developmental Toxicology, National Institutes of Health

111 T.W. Alexander Dr. Research Triangle Park, NC 27709 (USA)

Tel. +1 919541 1095, Fax +1 919541 3800, E-Mail mishina@niehs.nih.gov 


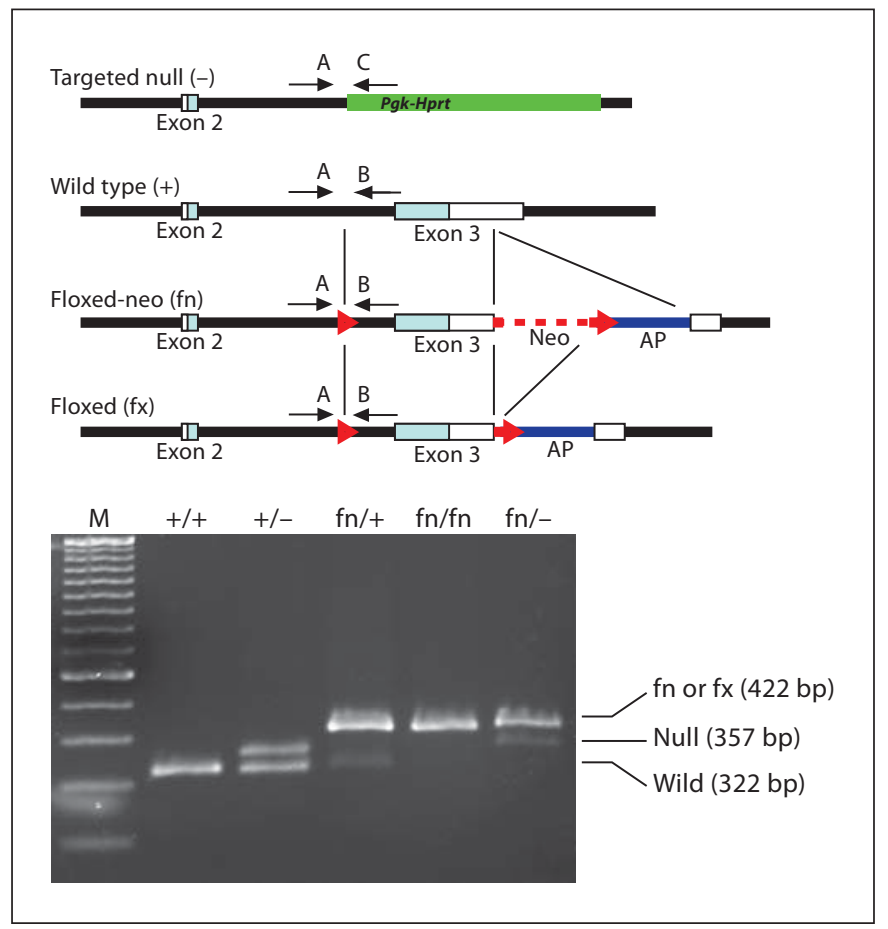

Fig. 1. Schematic representation of different alleles of the $B m p 2$ locus. A loxP site (red triangle) was inserted in intron 2 and a polII-neo cassette flanked by 2 FRT sites (red squares) followed by the other loxP site and human alkaline phosphatase gene (blue line) was inserted into the $3^{\prime}$ untranslated region of exon 3 (white box). Coding regions in exons 2 and 3 are highlighted as light blue. Gene targeting in ES cells resulted in the floxed-neo $(f n)$ allele. The neo cassette was removed by breeding with an FLPe mouse line to generate the floxed allele $(f x)$. Structure of the conventional null allele [Zhang and Bradley, 1996] is also shown. Examples of PCR genotyping are shown in the lower panel. M; 100 bp ladder.

(UTR) of Bmp2 as a selection marker for gene targeting events (manuscript in preparation and fig. 1).

It is known that the presence of a neo selection cassette in the targeted locus sometimes compromises gene function, generating a hypomorphic allele [Olson et al., 1996; Meyers et al., 1998]. In the course of evaluating the gene activity of the floxed allele for $B m p 2$ with or without the neo cassette, we found that the embryos genotyped as floxed with the neo cassette over the conventional null allele $(f n /-)$ were at a disadvantage for survival, and this phenomenon depended on the genotype of the mother. These results suggest that the appropriate levels of BMP2 in embryos are critical for their normal development and the levels of Bmp2 expression from mothers also influence the penetrance of these abnormalities when combined with lower BMP2 levels in the embryo.

\section{Materials and Methods}

\section{Mouse Strains}

Detailed description for generation of a floxed allele of $B m p 2$ will be provided elsewhere (manuscript in preparation). In brief, a polII-neo cassette flanked by FRT sites followed by one loxP site, a splicing acceptor and human alkaline phosphatase expression cassette was introduced into the $3^{\prime}$-UTR of exon 3 of Bmp2 via homologous recombination in embryonic stem (ES) cells. The resulting allele was designated as floxed-neo ( $f n$ ) (fig. 1). After germline transmission of the targeted allele, heterozygous mice for the $f n$ allele were bred with FLPe mice [Farley et al., 2000] to remove the neo selection cassette. This allele was designated floxed ( $f x$ ) (fig. 1). Mice carrying a conventional null allele were obtained from Allan Bradley [Zhang and Bradley, 1996]. These mouse lines were maintained in mixture of $129 \mathrm{SvEv}$ and C57BL6/J background. All animal experiments were conducted according to the U.S. Public Health Service policy on the humane care and use of animals. All animal procedures used in this study were approved by the National Institute of Environmental Health Sciences Institutional Animal Care and Use Committee.

\section{Genotyping}

Genotypes of the embryos and pups were determined by PCR analyses of genomic DNA extracted from amnion of embryos or from ear punch of adults, with the following set of primers: A: $5^{\prime}$ AGCATGAACCCTCATGTGTTGG-3'， B: 5'-GTGACATTABBCTGCTGTAGCA-3', and C: 5'-GAGACTAGTGAGACGTGCTACT-3'. Positions of the primers and examples of the genotyping results are shown in figure 1 . Sizes of the PCR products are 322 bp for the wild type, $357 \mathrm{bp}$ for the null (-) allele, $422 \mathrm{bp}$ for $f n$ and $f x$ alleles. The $f x$ allele was differentiated from the $f n$ allele with primers amplifying between exon 3 and human alkaline phosphatase; 5'-AGGGTTTCAGGTCAGTTTCCG-3' and 5'-GATGATGAGGTTCTTGGGCGG-3' (450 bp for $f x$ allele, $2 \mathrm{~kb}$ for $f n$ allele).

\section{Quantitative PCR}

For embryonic samples, pregnant mice were sacrificed at E9.5 and extra-embryonic and embryonic tissues were dissected. The extra-embryonic tissues were used for subsequent genotyping and the embryos were surgically divided into anterior and posterior regions. For maternal samples, pregnant mice were sacrificed at E6.5, and uteri and deciduas were removed. RNA was isolated from these tissues using TRIzol (Invitrogen) and cDNA was synthesized using the SuperScript First-Strand Synthesis System (Invitrogen) and random hexamer primers. Real-time PCR measurements of individual cDNAs were performed with the ABI Prism 7700 sequence detection system. Gene-specific primers for Bmp2 (Mm01340178_m1) were purchased as the pre-designed TaqMan gene expression assays gene-specific probe and primer mixture (PE Applied Biosystems). The TaqMan rodent glyceraldehyde-3-phosphate dehydrogenase (Gapdh) control reagent (PE Applied Biosystems) was used as an internal control. All measurements were performed in triplicate. Values were normalized to Gapdh using the $2^{-\Delta \Delta \mathrm{Ct}}$ method and expressed as means $\pm \mathrm{SD}$. Statistical differences were determined by a Student's $t$ test. Statistical significances are relative to the corresponding wild type control animals. 


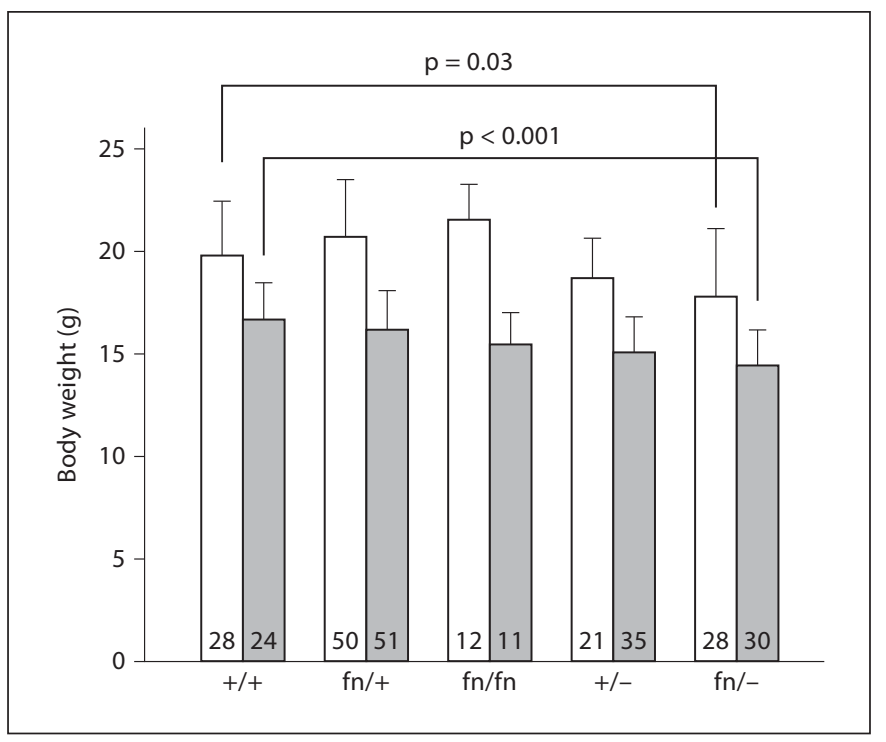

Fig. 2. Reduction of body weight in $f n$ mice. Body weight was measured at 38 days after birth. Numbers at the bottom of each bar represent the total number of mice that were measured. Open bar $=$ male; shaded bar $=$ female. Student's t test was done for statistical analyses.

\section{Results}

Floxed-Neo (fn) Allele for Bmp2 is Disadvantageous for Survival

To address whether the presence of the neo cassette in the Bmp2 locus compromises its function, we first set up the breeding between heterozygous mutant mice for the floxed-neo $(f n)$ allele and heterozygous mutant mice for conventional null allele to generate pups transheterozygous for $f n$ and null allele for $B m p 2(f n /-)$. In this case, 4 different genotypes of pups with the same ratio were expected. However, individuals whose genotype was $f n /-$ were underrepresented (table 1). The proportion of $f n /-$ pups was more severely underrepresented when mothers were heterozygous for null (+/-) versus heterozygous for floxed-neo $(f n /+)$ (table 1). Body weight of the surviving pups of the genotype $f n /-$ at 38 days after birth was significantly lower than that of the wild type littermates (fig. 2). Many of the $f n /$ - individuals had kinked tails (fig. 3A, B) and this phenotype was already evident during embryogenesis (fig. 3C-E). When fathers homozygous for the floxed allele without the neo cassette $(f x / f x)$ were bred with heterozygous null females (+/-), we found the expected ratio of genotypes of pups (table 1). These findings prompted us to hypothesize that the $f n$ allele, but not the $f x$ allele, was functionally hypomorphic; and
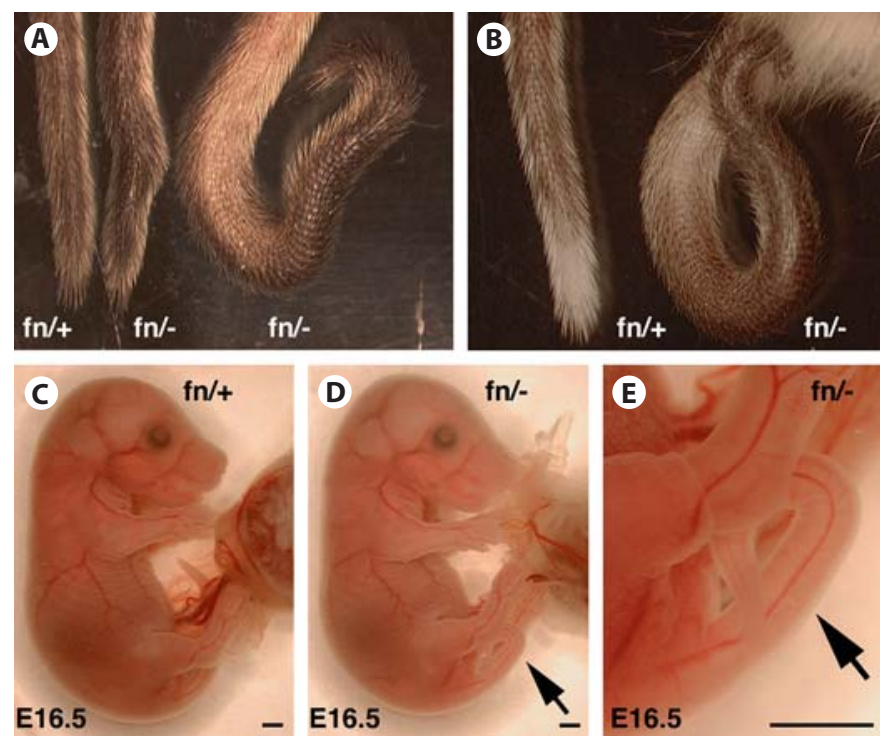

fn/-

Fig. 3. Kinky tail phenotype. A, B Three week old $f n /-$ mice showed kinky tails with varying degree. Tails from control littermates $(f n /+)$ are also shown. D, E The kinky tail phenotype (arrows) develops during embryogenesis. E16.5 $\mathrm{fn} /$ - embryos that developed the phenotype in an $f n /+$ mother are shown. C Control. Scale bar $=1 \mathrm{~mm}(\mathbf{C}-\mathbf{E})$.

Table 1. Underrepresentation of $f n /-$ pups

\begin{tabular}{|c|c|c|c|c|c|}
\hline \multicolumn{2}{|c|}{ Genotype of } & \multicolumn{4}{|c|}{ Number of pups for each genotype (\%) } \\
\hline Father & Mother & $+/+$ & $+/-$ & $f n /+$ & $f n /-$ \\
\hline$+1-$ & $f n /+$ & $22(25)$ & $20(23)$ & $35(41)$ & $10(11)$ \\
\hline$f n /+$ & $+/-$ & $23(28)$ & $36(43)$ & $20(24)$ & $4(5)$ \\
\hline$+1-$ & $f n / f n$ & - & - & $59(65)$ & $32(35)$ \\
\hline$f n / f n$ & $+1-$ & - & - & $100(81)$ & $23(19)$ \\
\hline Father & Mother & $+/+$ & $+/-$ & $f x /+$ & $f x /-$ \\
\hline$f x / f x$ & $+/-$ & - & - & $26(53)$ & $23(47)$ \\
\hline
\end{tabular}

more importantly, a lowered BMP2 level in the mother has an influence during pregnancy when combined with the reduced levels of BMP2 in the embryo. Various types of breeding and analysis were set up to test this idea.

If the $f n$ allele is hypomorphic, then the mice with the genotype of $f n$ over the conventional null $(f n /-)$ should exhibit more severe phenotypes than the conventional heterozygous mutants (+/-). The mating schedule shown in table 2 was set up to determine whether distortion of the genotype ratio would become more prominent, if 
Table 2. Underrepresentation of $f n /$ - pups

\begin{tabular}{|c|c|c|c|c|c|c|}
\hline \multicolumn{2}{|c|}{ Genotype of } & \multicolumn{5}{|c|}{ Number of pups for each genotype (\%) } \\
\hline Father & Mother & $+/-$ & $-1-$ & $f n /+$ & $f n /-$ & $f n / f n$ \\
\hline$f n /-$ & $+/-$ & $14(41)$ & $0(0)$ & $15(45)$ & $5(14)$ & - \\
\hline$+/-$ & $f n /-$ & $21(36)$ & $0(0)$ & $25(44)$ & $12(20)$ & - \\
\hline$f n /-$ & $f n /-$ & - & $0(0)$ & - & $25(47)$ & $28(53)$ \\
\hline Father & Mother & $+/-$ & $-1-$ & $f x /+$ & $f x /-$ & $f x / f x$ \\
\hline$f x /-$ & $f x /-$ & - & $0(0)$ & - & $42(63)$ & $24(36)$ \\
\hline
\end{tabular}

mothers' genotypes were $f n /-$. As shown in table 2 , the proportion of $f n /-$ pups at weaning stage was lower than expected $(33 \%)$ in both the $+/$ - mothers and $f n /$ - mothers ( $14 \%$ and $20 \%$, respectively). However, the ratio of $f n /-$ pups in the litters from $f n /$ - mothers was not drastically reduced compared with that from $+/-$ mothers. In the case of intercross between $\mathrm{fn} /$ - mice, $47 \% \mathrm{fn} /$ - individuals reached weaning stage instead of the expected $67 \%$ (the expected ratio of $f n / f n: f n /-:-/-$ at weaning stage is 1:2:0). In the case of an intercross of $f x /$ - mice, the expected ratio of $f x /$ - individuals was obtained at the weaning stage (table 2), supporting the idea that the neo cassette influenced embryo survival. Interestingly, intervals of conception to delivery for $f n /$ - females were slightly longer than those of $+/$ - females $(27.5 \pm 6.4$ days, $n=11$ and $22.8 \pm 1.6$ days, $\mathrm{n}=21$, respectively), while litter size was dramatically reduced $(3.9 \pm 2.2, \mathrm{n}=9$ and $6.2 \pm 1.9$, $\mathrm{n}=14$, respectively, $\mathrm{p}<0.01$ ). The average litter size for wild type females over the past several years in our colony with similar genetic background is 7.3. These results reinforce the idea that the $f n$ allele, not the $f x$ allele, is hypomorphic, and suggest that reduced BMP2 function in $\mathrm{fn} /$ - mothers compromises survival of embryos regardless of the genotype of embryos.

\section{Floxed-Neo (fn) Allele for Bmp2 Causes Closure \\ Defects in Cephalic Neural Tube and Ventral \\ Abdominal Body Wall}

To identify the cause(s) of lethality, we set up timed matings in several combinations of the genotypes for parents. As previously reported, homozygous null mice were morphologically distinguished as early as embryonic day 8.5 (E8.5) [Zhang and Bradley, 1996] due to their defects in embryonic turning and neural tube closure at E9.5 (fig. 4A). All $f n /-$ embryos turned by E9.5, but some of them showed closure defects of the cephalic neural tube
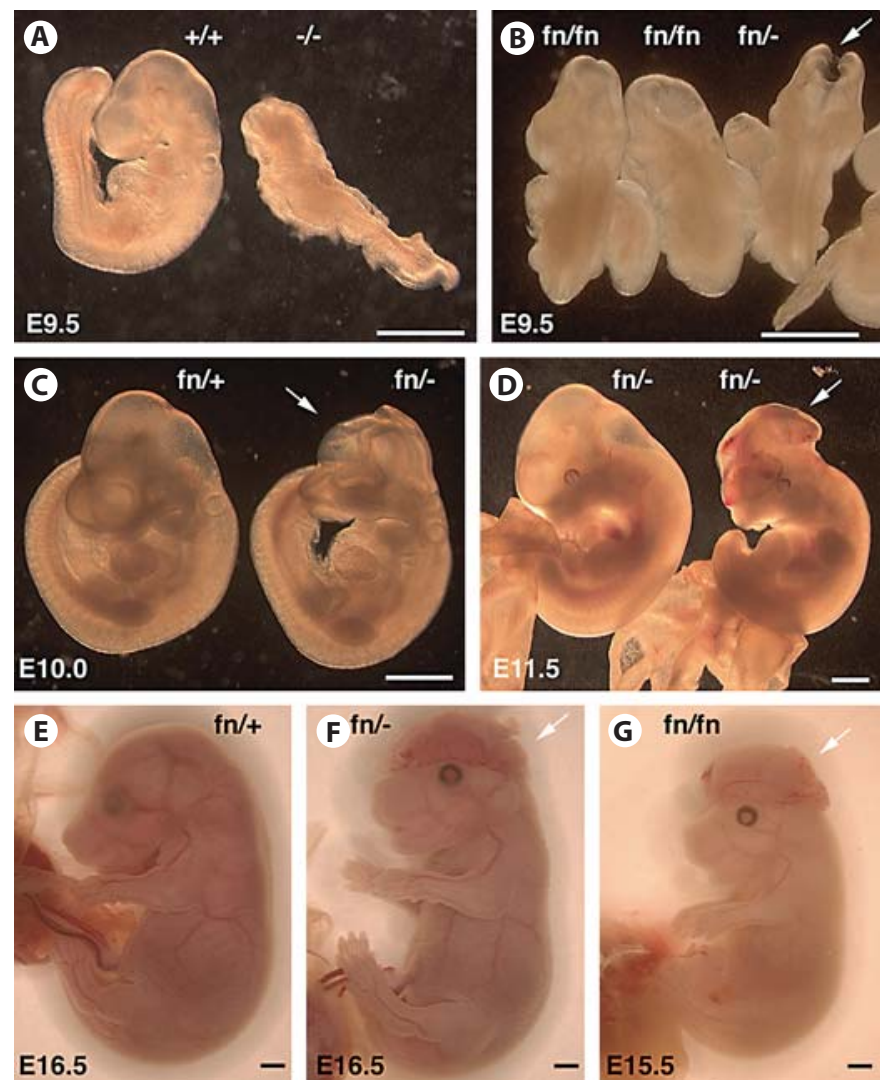

Fig. 4. Neural tube closure defects. A At E9.5, homozygous null mutant embryos (-/-) were distinct by their defects in turning and neural tube closure along the body axis. B Some of the $\mathrm{fn} /-$ embryos showed closure defects in the cephalic neural tube at E9.5 (white arrow). The closure defects persisted during embryogenesis, but were limited to the cephalic region and resulted in exencephaly as shown in E10.0 (C), E11.5 (D), E16.5 (E, F). Some of the $f n / f n$ embryos from $f n /-$ mothers developed similar closure defects $(\mathbf{G})$. Genotype of mothers is $+/-(\mathbf{A}, \mathbf{C}, \mathbf{E}, \mathbf{F}), f n /+(\mathbf{B})$, and $f n /-(\mathbf{D}, \mathbf{G})$. Scale bar $=1 \mathrm{~mm}$.

(fig. 4B, C) and developed exencephaly during mid-gestation (fig. 4D, F). Frequency of the closure defects for the neural tube in $f n /$ - embryos strongly depended on the genotype of the mothers (table 3). For example, if the mother was $f n / f n,+/-$, or $f n /-$, the frequency of neural tube defects in $f n /-$ embryos was $\sim 25 \%$, but if the mother was $f n /+$, the frequency was $15 \%$ in the $f n /-$ embryos (table 3). Two of the $f n / f n$ embryos out of 27 collected from $f n /-$ mothers showed similar types of closure defects (fig. $4 \mathrm{G}$ ), while none of the $f n / f n$ embryos collected from $f n /+$ and $f n / f n$ mothers $(n=36)$ showed the defects. These facts again demonstrate that a reduction of BMP2 in the mother affects the development of the neural tube closure defects in $f n / f n$ embryos. 

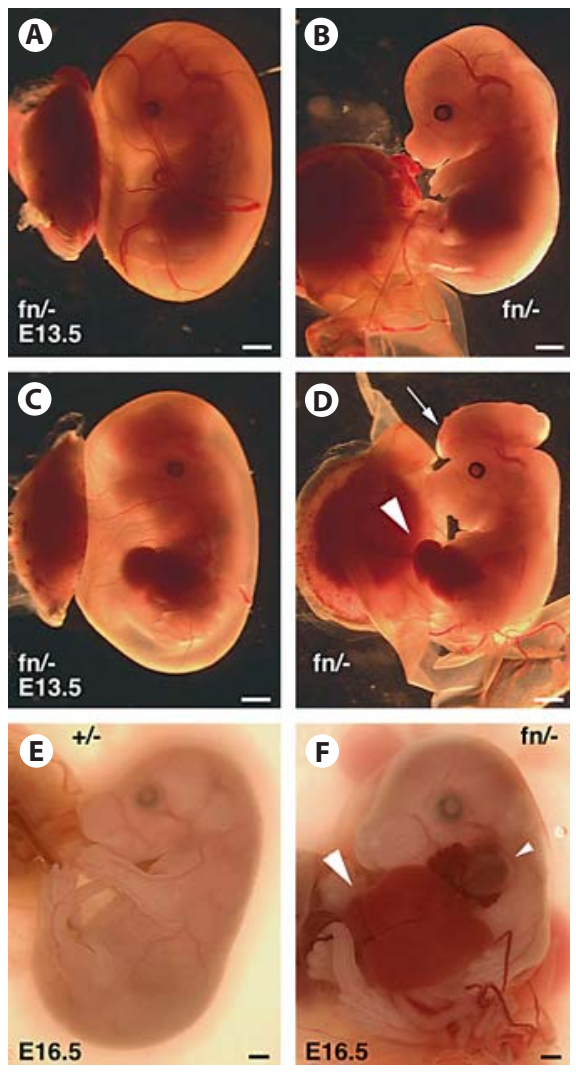

Fig. 5. Body wall closure defects found in the ventral abdominal region of $f n /$ - embryos. A-D Two $f n /-$ embryos from the same litter at E13.5. One looked normal (A, B) and the other (C, D) showed body wall closure defects (hernia, white arrowhead) along with exencephaly (white arrow). Gross morphology before $(\mathbf{A}, \mathbf{C})$ and after (B, D) removal of the yolk sac is shown. E Normal littermate. F Some of the $f n /-$ embryos at E16.5 showed only the body wall closure defect (arrowheads), but not exencephaly. Genotypes of mothers for these embryos are $+/-$. Scale bar $=1 \mathrm{~mm}$.

Some of the $f n /-$ embryos also developed a body wall hernia in the ventral abdominal region (fig. 5A-D). In many cases, this abnormality coincided with the defects in neural tube closure, but not always (fig. 5F). As shown in table 3, frequency of this phenotype also varied among the genotypes of mothers with the highest frequency in $f n /-$ embryos when the mother was heterozygous for conventional null allele (+/-). Although the numbers and frequency are small for this analysis, they clearly suggest a maternal influence on development of this abdominal abnormality.

\section{Expression Level of the Floxed-Neo Allele is Lower}

The direct hypothesis is that the amount of $B m p 2$ transcripts generated from the $f n$ allele is lower due to the
Table 3. Abnormalities found in $f n /-$ embryos

\begin{tabular}{llrlll}
\hline \multirow{2}{*}{ Mother } & \multicolumn{4}{l}{ Number of abnormal embryos (\%) } \\
\cline { 2 - 6 } & Total & NTD & Hernia & Kinked & Dying \\
\hline$f n /+$ & 13 & $2(15)$ & $0(0)$ & $2(15)$ & $1(8)$ \\
$f n / f n$ & 31 & $7(23)$ & $1(3)$ & $1(3)$ & $0(0)$ \\
$+/-$ & 44 & $11(25)$ & $6(14)$ & $6(14)$ & $1(2)$ \\
$f n /-$ & 17 & $4(24)$ & $1(6)$ & $3(18)$ & $2(12)$ \\
\hline
\end{tabular}

NTD: Neural tube closure defects.

presence of the neo cassette in the 3'-UTR. To address this issue, we extracted RNA from embryos at E9.5 and uteri at E6.5 for quantification of $B m p 2$ transcript levels. RNA was also extracted from decidual tissues at E6.5 since $B m p 2$ is known to be highly expressed in the decidua around this stage and believed to play critical roles for the establishment of implantation and subsequent embryogenesis [Ying and Zhao, 2000; Paria et al., 2001; Li et al., 2007]. As expected, expression levels of Bmp2 in homozygous embryos for the $f n$ allele were dramatically reduced both in the anterior and posterior halves compared to those of wild type (fig. 6A, B; 6\% and 7\%, respectively). Expression levels of $B m p 2$ in $f n / f n$ embryos were much lower than those of heterozygous null embryos (fig. 6A, B, 17\% for the anterior half and 10\% for the posterior half relative to the wild type). Expression levels of $B m p 2$ in $f n /-$ embryos were slightly lower than those of $f n / f n$ embryos (fig. 6A, B, 5\% for the anterior half and $4 \%$ for the posterior half). Results from both uterus and decidua showed the same expression profile as embryos (fig. 6C, D). Expression levels of Bmp2 in decidua and uterus in $f n /+$ were $70 \%$ and $68 \%$, respectively, while in mice heterozygous for the conventional null allele they were $30 \%$ and $24 \%$, respectively. These results suggest that the different degree of underrepresentation of $f n /-$ pups between $\mathrm{fn} /+$ mothers and $+/-$ mothers $(11 \%$ and $5 \%$, respectively) is due to the difference in expression of $B m p 2$ in the maternal tissues adjacent to the embryos. However, expression levels of $B m p 2$ in the maternal tissues in $f n / f n$ individuals were similar to or lower when compared to those of $+/-$ individuals (32\% vs. $30 \%$ in decidua and $10 \%$ vs. $24 \%$ in uterus), suggesting that there could be more complex reasons to explain the difference shown in table 1 for the expected number of pups with the various genotypes. As expected from the breeding results, expression levels of $B m p 2$ in $f x / f x$ mothers were comparable to those in the wild type (fig. 6C, D, 93\% in 


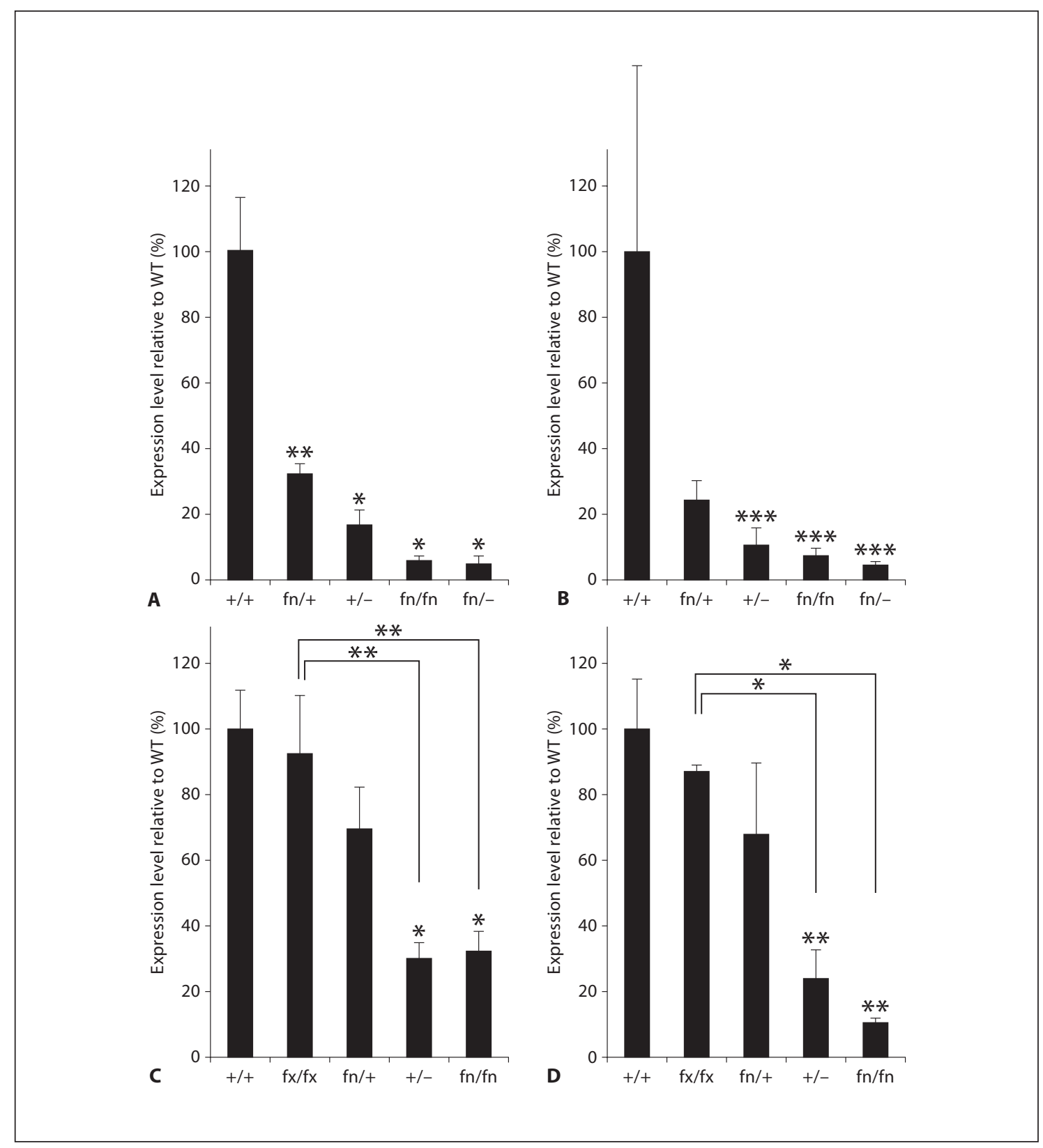

Fig. 6. Lowered expression of $B m p 2$ from the $f n$ allele. Expression levels of $B m p 2$ were measured by quantitative RT-PCR and their relative levels against that of the wild type are shown. Averages of at least 3 independent samples are shown with standard deviation. A E9.5 embryos, anterior half. B E9.5 embryos, posterior half. C Decidual tissues at E6.5. D Uterus at E6.5. Genotypes are shown at the bottom of each bar. Student's t test was done for statistical analyses. ${ }^{*} \mathrm{p}<0.025 ;{ }^{* *} \mathrm{p}<0.05 ;{ }^{* *} \mathrm{p}<0.1$.

decidua and $87 \%$ in uterus) and significantly higher than those in $f n / f n$ mothers, reinforcing the idea that presence of the neo cassette, not loxP sites or the human alkaline phosphatase cassette in the $3^{\prime}-\mathrm{UTR}$, makes the Bmp2 allele hypomorphic.

Influence of BMP2 Levels on Mouse Embryo Development

\section{Discussion}

Here we showed that the presence of the neo cassette in the 3'-UTR of Bmp2 lowered the expression level of this gene, and caused abnormalities in adults, including low-

Sex Dev 2008;2:134-141 
er body weight and a kinked tail. Lowered expression of Bmp2 also caused an abnormal embryonic phenotype, including neural tube closure defects and body wall closure defects, leading to lethality. Interestingly, genotypes of mothers influenced the frequency of these abnormalities in embryos. When there were lower levels of maternal $B m p 2$ expression in the uterus and/or decidua, higher frequencies of embryonic defects were observed in embryos with already reduced BMP2 levels. These abnormalities were rescued after removal of the neo cassette from the locus that led to resumed levels of normal Bmp2 expression.

All embryos homozygous for the conventional null allele (-/-) show abnormalities, including defects in turning, defects in neural tube closure, and failure to develop limb buds (fig. 4) [Zhang and Bradley, 1996]. Recently, we found that a portion of the heterozygous embryos for the conventional null allele (+/-) also developed neural tube closure defects in cephalic regions, in part depending on strain background [Castranio and Mishina, submitted]. These abnormalities found in $+/-$ embryos resembled those shown in this study (fig. 4). In both cases, closure defects in the trunk region were never observed, suggesting that the cephalic region of the neural tube is much more sensitive to BMP2 levels than the trunk region. It is proposed that morphological changes of the neural plate to form paired dorsolateral hinge points (DLHPs) are necessary for neural tube closure at the trunk level [Copp et al., 2003]. Recently it has been reported that antagonisms of BMP2 stimulate neural tube closure in the trunk region through formation of DLHPs [Ybot-Gonzalez et al., 2007]. However, in the cephalic region the formation of DLHPs is not required for neural tube closure [YbotGonzalez et al., 2002; Copp, 2005]. Together with our results in this study, these reports support the idea that BMP2 plays an important role in neural tube closure, presumably with different mechanisms or thresholds of BMP2 required between cephalic and trunk regions. Interestingly, frequencies of the neural tube defects in the cephalic region of $f n /-$ embryos were dramatically increased when the genotypes of the mothers are $f n / f n$, $+/-$, or $f n /$ - compared to $f n /+$ (table 3 ), suggesting that maternal factors significantly contribute to the frequency of the defects. It is still an unanswered question whether maternal BMP2 is directly involved in the closure process of embryos. It is possible that downstream target genes of BMP2 signaling, either in maternal tissues or embryos, are associated with this closure process.

We also found that some of the $f n /-$ embryos developed closure defects in ventral body wall resulting in ab- dominal hernia (fig. 5). This phenotype resembles a human condition known as omphalocele [Brewer and Williams, 2004; Wilson and Johnson, 2004]. We recently reported that mesoderm-derived tissue-specific disruption of Bmprla, a receptor for BMPs including BMP2, results in an omphalocele-like defect [Sun et al., 2007]. It has also been reported that double homozygous mutant embryos for $M s \times 1$ and $M s \times 2$, known downstream target genes of BMP signaling, develop similar defects [Ogi et al., 2005]. These results suggest that lowered expression of $B m p 2$ would decrease the expression levels of its downstream targets, including $M s \times 1$ and $M s \times 2$, and cause a failure of proper closure of the ventral abdominal wall. We have never observed body wall closure defects in over 1,000 Bmp 2 +/- embryos dissected over 10 years (unpublished results), suggesting that proper development of ventral abdominal body wall requires less BMP2 than that of cephalic neural tube closure.

Bmp2 is highly expressed in decidual tissues as well as in the lumen of the uterus soon after implantation [Ying and Zhao, 2000; Paria et al., 2001; Li et al., 2007]. Recent studies using a conditional gene disruption technology have revealed that BMP2 from the maternal side plays a role during implantation and subsequent embryogenesis. A uterus epithelium-specific disruption of $B m p 2$ results in failure of decidua formation and implantation [Lee et al., 2007]. Smaller litter size from $f n /-$ females could be explained by this fact, as the levels of BMP2 in uterus and/or decidua of $f n /-$ females are too low, and thus cannot fully support the development of embryos regardless of their genotype. Indeed, we observed an increase in the number of resorption sites in $f n /$ - mothers when dissected at early to mid-gestation (data not shown). It is notable that among the surviving embryos in $f n /$ - mothers, some of the $f n / f n$ embryos develop neural tube closure defects providing more supporting evidence that expression levels of Bmp2 in mothers have an impact on development of abnormalities in these embryos.

The remaining question is how the presence of the neo cassette is negatively influencing the expression level of $B m p 2$. It is believed that the $3^{\prime}$-UTR plays a role in degradation of mRNA, providing a binding site for enzymes for RNA degradation [Jacobson and Peltz, 1996; Blackshear, 2002]. There is accumulating evidence that alteration of the 3'-UTR changes steady state levels of transcripts. Therefore, it is reasonable to speculate that presence of the neo cassette in the 3'-UTR would stimulate degradation of the $B m p 2$ transcript. Alternatively, but not exclusively, presence of the strong polII promoter may influence regulation, transcription or splicing of the Bmp2 
gene. It is very intriguing that after removal of the neo cassette from the Bmp2 locus ( $f x$ allele), expression levels of $B m p 2$ become comparable to the wild type (fig. 6C, D), despite the presence of the human alkaline phosphatase cassette in the 3'-UTR of Bmp2. This implies that not only the location, but also the sequence of the neo cassette or the polII promoter alter regulation and levels of $B m p 2 \mathrm{ex}-$ pression. Recent SNP analyses in humans have revealed that there are 6 SNPs located in the $3^{\prime}$-UTR of BMP2 (http://www.genecards.org/cgi-bin/carddisp.pl?gene=B MP2\&search=bmp2). At this moment, no correlation of these SNPs with peculiar phenotypes is reported. However, one of the SNPs, rs15705, results in disruption of a putative posttranscriptional regulatory motif [Fritz et al.,
2006]. Together with the results found in this study, these data suggest that the $3^{\prime}$-UTR of the Bmp2 locus controls the amount of Bmp2 transcripts and alteration of this 3'UTR function may result in embryonic abnormalities.

\section{Acknowledgements}

We gratefully thank Drs. Hongbing Zhang and Allan Bradley for Bmp2 conventional null mice. We thank Ms. Leigh E. Davis, Ijeoma Nwosu, Gloria MacDonald, and Kelly McCann for their technical support, Ms. Tonya Miller for her excellent service of maintenance of the mouse colonies. This work was supported by the Intramural Research Program of the NIEHS/NIH to Y.M. (ES071003-10).

\section{References}

Blackshear PJ: Tristetraprolin and other CCCH tandem zinc-finger proteins in the regulation of mRNA turnover. Biochem Soc Trans 30:945-952 (2002).

Brewer S, Williams T: Finally, a sense of closure? Animal models of human ventral body wall defects. Bioessays 26:1307-1321 (2004).

Copp AJ: Neurulation in the cranial region normal and abnormal. J Anat 207:623-635 (2005).

Copp AJ, Greene ND, Murdoch JN: The genetic basis of mammalian neurulation. Nat Rev Genet 4:784-793 (2003).

Correia AC, Costa M, Moraes F, Bom J, Novoa A, Mallo M: Bmp2 is required for migration but not for induction of neural crest cells in the mouse. Dev Dyn 236:2493-2501 (2007).

$\checkmark$ de Caestecker M: The transforming growth factor-beta superfamily of receptors. Cytokine Growth Factor Rev 15:1-11 (2004).

-Farley FW, Soriano P, Steffen LS, Dymecki SM: Widespread recombinase expression using FLPeR (flipper) mice. Genesis 28:106-110 (2000).

Fritz DT, Jiang S, Xu J, Rogers MB: A polymorphism in a conserved posttranscriptional regulatory motif alters bone morphogenetic protein 2 (BMP2) RNA:protein interactions. Mol Endocrinol 20:1574-1586 (2006).

- Jacobson A, Peltz SW: Interrelationships of the pathways of mRNA decay and translation in eukaryotic cells. Annu Rev Biochem 65:693739 (1996).

-Kanzler B, Foreman RK, Labosky PA, Mallo M: BMP signaling is essential for development of skeletogenic and neurogenic cranial neural crest. Development 127:1095-1104 (2000).
Kishigami S, Mishina Y: BMP signaling and early embryonic patterning. Cytokine Growth Factor Rev 16:265-278 (2005).

Lee KY, Jeong JW, Wang J, Ma L, Martin JF, et al: Bmp2 is critical for the murine uterine decidual response. Mol Cell Biol 27:5468-5478 (2007).

- Li Q, Kannan A, Wang W, Demayo FJ, Taylor $\mathrm{RN}$, et al: Bone morphogenetic protein 2 functions via a conserved signaling pathway involving Wnt4 to regulate uterine decidualization in the mouse and the human. J Biol Chem 282:31725-31732 (2007).

- Ma L, Lu MF, Schwartz RJ, Martin JF: Bmp2 is essential for cardiac cushion epithelialmesenchymal transition and myocardial patterning. Development 132:5601-5611 (2005).

Meyers EN, Lewandoski M, Martin GR: An Fgf8 mutant allelic series generated by Cre- and Flp-mediated recombination. Nat Genet 18: 136-141 (1998).

Ogi H, Suzuki K, Ogino Y, Kamimura M, Miyado $\mathrm{M}$, et al: Ventral abdominal wall dysmorphogenesis of $M s \times 1 / M s x 2$ double-mutant mice. Anat Rec A Discov Mol Cell Evol Biol 284:424-430 (2005).

Olson EN, Arnold HH, Rigby PW, Wold BJ: Know your neighbors: three phenotypes in null mutants of the myogenic bHLH gene MRF4. Cell 85:1-4 (1996).
Paria BC, Ma W, Tan J, Raja S, Das SK, et al: Cellular and molecular responses of the uterus to embryo implantation can be elicited by locally applied growth factors. Proc Natl Acad Sci USA 98:1047-1052 (2001)

Sun J, Liu YH, Chen H, Nguyen MP, Mishina Y, et al: Deficient Alk3-mediated BMP signaling causes prenatal omphalocele-like defect. Biochem Biophys Res Commun 360:238243 (2007).

Tsuji K, Bandyopadhyay A, Harfe BD, Cox K, Kakar S, et al: BMP2 activity, although dispensable for bone formation, is required for the initiation of fracture healing. Nat Genet 38:1424-1429 (2006)

Wilson RD, Johnson MP: Congenital abdominal wall defects: an update. Fetal Diagn Ther 19: 385-398 (2004).

Ybot-Gonzalez P, Cogram P, Gerrelli D, Copp AJ: Sonic hedgehog and the molecular regulation of mouse neural tube closure. Development 129:2507-2517 (2002).

Ybot-Gonzalez P, Gaston-Massuet C, Girdler G, Klingensmith J, Arkell R, et al: Neural plate morphogenesis during mouse neurulation is regulated by antagonism of Bmp signalling. Development 134:3203-3211 (2007)

Ying Y, Zhao GQ: Detection of multiple bone morphogenetic protein messenger ribonucleic acids and their signal transducer, Smad1, during mouse decidualization. Biol Reprod 63:1781-1786 (2000).

-Zhang H, Bradley A: Mice deficient for BMP2 are nonviable and have defects in amnion/chorion and cardiac development. Development 122:2977-2986 (1996). 